\title{
Significance of N-Terminal Pro Brain Natriuretic Peptide as a Diagnostic Tool for Premature Ventricular Contractions in Patients with Preserved Left Ventricular Function"
}

Shazadi Ambreen ( $\square$ shazadiambreen@hotmail.com )

Shifa Tameere Millat University,Islamabad

Humaira Fayyaz khan

Riphah International University, Rawalpindi

Azmat Hayat

Armed forces institute of cardiology and heart diseases (AFICD)Rawalpindi Pakistan

Shazia Ali

Riphah International University, Rawalpindi

\section{Research Article}

Keywords: PVCs, PVCs burden, BNP, NT-proBNP, Cardiomyopathy

Posted Date: March 10th, 2021

DOl: https://doi.org/10.21203/rs.3.rs-263047/v1

License: (c) (i) This work is licensed under a Creative Commons Attribution 4.0 International License.

Read Full License 


\section{Abstract}

\section{Objective}

The study was aimed to evaluate the levels of NT-proBNP in mild, moderate and frequent ventricular ectopic burden in patients with preserved left ventricular function.

\section{Material and methods}

A case control study was conducted in Islamic International Medical College (IIMC) in collaboration with Armed Forces Institute of Cardiology (AFIC) after approval from ethical review committee (ERC) of Riphah University (Ref \#Riphah/IIMC/ERC/17/0230). It comprised of 120 Premature ventricular contractions (PVCs) patients with 40 patients in each group of mild ( $<10 \%)$, moderate $(10-20 \%)$ and frequent (>20\%) PVCs burden. 40 healthy subjects with no PVCs were taken as a control group. NT-proBNP levels were measured in all groups and statistical analysis was done by applying ANOVA and Pearson correlation tests to evaluate its association with PVCs burden

\section{Results}

NT-proBNP levels were found to be raised in all PVCs burden of $<10 \%, 10-20 \%$ and $>20 \%$ as compare to control group with $p$ value of $0.039,0.02$ and 0.009 respectively. $p$ value of $\leq 0.05$ was considered significant. Symptomatic and asymptomatic patients show higher NT-proBNP levels as compared to control group with p-value of .002 and .04.

\section{Conclusion}

It is concluded that NT-proBNP levels are raised in patients of PVCs. This finding can be helpful for diagnostic purposes of PVCs.

\section{Introduction}

Recently several new biomarkers have emerged as a more feasible and promising ways for patient stratification at high risk of cardiac events. Especially NT-proBNP, which is a B-type natriuretic peptide have both diagnostic and prognostic significance in congestive heart failure. ${ }^{1,2}$ Several studies have shown association of NT-proBNP with frequent PVCs (premature ventricular contractions) which are the extra beats generated in ventricles. ${ }^{3,4}$ Despite the fact that many studies have demonstrated the association of NT-proBNP with frequent PVCs, its importance as a diagnostic tool for PVCs still has to be evaluated. Thus we performed this case control cross sectional analytical study to evaluate levels of NTproBNP in all burdens of PVCs. This study is the first work according to our knowledge. A well-known complication of premature ventricular contractions is dilated type reversible PVCs induced cardiomyopathy which can occur in frequent PVCs as well as in patients with burden of $4 \%$. There is no criteria for stratifying these patients at high risk of developing cardiomyopathy. ${ }^{5}$ Thus this study aims to 
evaluate the NT-proBNP levels in all (mild, moderate and frequent) PVCs burdens providing a more feasible way for diagnosing and screening PVCs to prevent its complications.

\section{Materials And Methods \\ Study design:}

A case control cross sectional analytical study was conducted in Islamic International Medical College, Rawalpindi in collaboration with Electrophysiology department of Armed Forces during the period of $17^{\text {th }}$ September 2017 to $19^{\text {th }}$ May 2018 after approval from ethical review committee of Riphah University Islamabad (ERC). A total of 90 PVCs patients between the ages of 26-60 were included in the study from Armed forces institute of cardiology patients by probability convenient sampling. All the patients were included in the study after obtaining the informed consent. All methods were carried out in accordance with relevant guidelines and regulations for treating and managing the premature ventricular contractions in the patients. Patients coming with complaints of palpitations (anxiety was ruled out after psychological assessment), chest pain, heart sinking, and dizziness were evaluated for the presence of arrhythmias. Patients with Premature ventricular contractions (PVCs) were selected and the patients with arrhythmias other than PVCs were excluded from the study. Predefined criteria for the classification of PVCs into mild, moderate and frequent PVCs burden was used to include the equal ratio of PVCs burden.

\section{Patient's selection:}

All the patients were evaluated by physical examination, 12 -lead electrocardiography (ECG), 24 hours Holter monitoring and transthoracic echocardiography. Exercise tolerance test (ETT) was performed in all the patients. Further investigations such as multi-detector computed tomography, and MRI was done in some patients if indicated and the patients with structural heart disease and coronary artery disease were excluded from the study.

Exclusion criteria for the study included trauma, Physical impairment, pregnancy, Hypertension, severe coronary artery disease, and pneumonia ,renal failure and systolic heart failure (Ejection fraction $<50 \%$ ). Patients with PVCs caused by reversible causes such as electrolyte imbalance, infection or drugs were excluded. All the patients were evaluated and treated according to the proper protocols established in armed forces institute cardiology. In the end 160 patients in total were included in the study with 40 patients in each group of mild, moderate and frequent PVCs burden.

40 subjects as a control were selected from the population between the ages of 25 to 65 years. Control group included both males and females. All these patients were healthy with no history of diseases like hypertension, cardiovascular diseases, structural heart diseases etc. patients with normal BMI was included in the group. 


\section{Demographic data:}

Demographic data regarding age (years) sex, height $(\mathrm{cm})$, weight $(\mathrm{kg}), \mathrm{BMI}$ was entered in a questionnaire Performa.

\section{ECG and Holter analysis:}

Patients with complaints of palpitations, chest pain, syncope or even asymptomatic patients (who were found to have PVCs on 12 lead ECG during their routine visits) were monitored for duration of 24 hours by Holter device (12 lead) to document any arrhythmia on advice of their attending cardiologist.

For Holter monitoring, 12 chest leads were applied to their chest and they were sent home along with Holter machine. After 24 hours Holter was removed and attached to the computer to transfer ECG data. After reading 24 hours recording of ECG, patients with ventricular ectopy/PVCs were selected and their PVCs burden was calculated. PVCs were diagnosed on 24 hours Holter monitoring and their burden was calculated by dividing the number of PVCs by total number of analyzed beats in 24 hours. According to their PVCs burden, they were divided into 3 groups of mild ( $<10 \%)$, moderate $(10-20 \%)$ and high $(>20 \%)$ burden and then the equal ratio of all the PVCs patients were taken.

\section{Echocardiography:}

Echocardiography was done in all the patients by a cardiologist. Systolic ejection fraction and diastolic ejection fraction were calculated and the patient with systolic ejection fraction less than $50 \%$ were not included in study.

\section{Estimation of $\mathrm{N}$ Terminal pro BNP levels:}

Blood for measurement of NT-proBNP concentrations was collected by venipuncture in polyethylene therephthalate glycol (PET) Vacuette clot and were centrifuged within one hour. Serum samples were stored at $-80^{\circ} \mathrm{C}$ till all the samples were collected and NT-proBNP levels were measured later on. NTproBNP was measured by electro chemiluminescent immunoassay using Human NT-proBNP ELISA kit.

(Synonyms: E-EL-M0918 Catalog No: E-EL-H0902 96T Manufacturer: Elabscience)

\section{Statistical Analysis:}

Statistical analysis was done by using spss version 25 . As the data was normally distributed Continuous variables are summarized as the mean \pm standard deviation and discrete variables are described as counts with percentages. Comparisons of NT - proBNP levels of control group with mild, moderate and 
frequent PVCs burden was performed using parametric tests. One way Analysis of variance (ANOVA) was applied. For categorical data, the chi-square test was used. Correlations between parameters were tested by Pearson's correlation analysis

\section{Results}

\section{Baseline and clinical characteristics:}

Patient's characteristics are shown in table 1. Clinical data analysis is given in Table: 2 which shows that among all the PVCs patients, 44(73\%) were symptomatic while 32(27\%) were asymptomatic. This shows that most of the patients with PVCs developed symptoms and the number was significantly high as compare to asymptomatic patients with $p$ value of 0.00 . Among all the patients $72(60 \%)$ were males and $48(40 \%)$ were females representing that more males were having PVCs but the difference was insignificant with $p$ value of 0.121 .

Table 1

Patient's baseline characteristics 


\begin{tabular}{|c|c|c|c|}
\hline Characteristics & Groups & $\mathrm{n}(\%)$ & Burden \\
\hline \multicolumn{4}{|l|}{ Age(years) } \\
\hline & $<44$ & $26(43.3)$ & $14.6 \pm 9.13$ \\
\hline & $45-54$ & $12(20)$ & $19.01 \pm 8.03$ \\
\hline & $55-64$ & $14(23.3)$ & $16.5 \pm 11.55$ \\
\hline & $>65$ & $8(13.3)$ & $10.25 \pm 6.4$ \\
\hline \multicolumn{4}{|l|}{ Occupation } \\
\hline & Major Physical work & $17(28.3)$ & $16.95 \pm 8.99$ \\
\hline & Major mental work & $32(53.3)$ & $13.52 \pm 9.56$ \\
\hline & Retired life & 11(18.3) & $18.02 \pm 9.27$ \\
\hline \multicolumn{4}{|l|}{ History of PVCs } \\
\hline & $<1 \mathrm{yr}$ & 11(18.3) & $16.37 \pm 8.44$ \\
\hline & $1-3$ yrs & $30(50.0)$ & $14.43 \pm 9.05$ \\
\hline & $>3$ yrs & 19(31.6) & $16.11 \pm 10.7$ \\
\hline \multicolumn{4}{|l|}{ Smoking } \\
\hline & Never/seldom & $5(8.3)$ & $24.25 \pm 8.04$ \\
\hline & Always & $47(78.3)$ & $15.91 \pm 9.09$ \\
\hline & Quit & $8(13.3)$ & $6.21 \pm 3.36$ \\
\hline \multicolumn{4}{|c|}{ Caffeinated beverages } \\
\hline & Never & 11(18.3) & $14.9 \pm 8.1$ \\
\hline & Low intake & $8(13.3)$ & $12.76 \pm 8.5$ \\
\hline & Medium intake & $29(48.3)$ & $15.30 \pm 9.6$ \\
\hline & High intake & 12(20) & $17.38 \pm 11.2$ \\
\hline
\end{tabular}


Table 2

Clinical characteristics

\begin{tabular}{|c|c|c|c|c|c|c|}
\hline $\begin{array}{l}\text { Demographic } \\
\text { and clinical } \\
\text { characteristics }\end{array}$ & $\begin{array}{l}\text { PVCs } \\
\text { cases, } \\
n=120(\%)\end{array}$ & $\begin{array}{l}\mathrm{p}- \\
\text { value }\end{array}$ & $\begin{array}{l}\text { Mean NT- } \\
\text { proBNP(ng) } \\
\text { in PVCs } \\
\text { patients }\end{array}$ & $\begin{array}{l}\text { p-value } \\
\text { (Comparing } \\
\text { NT-proBNP } \\
\text { levels in } \\
\text { gender, } \\
\text { symptoms } \\
\text { and burdens) }\end{array}$ & $\begin{array}{l}\text { Mean NT- } \\
\text { proBNP(ng) } \\
\text { in control } \\
\text { group } \\
\quad(n=40)\end{array}$ & $\begin{array}{l}\mathrm{p} \text { - value } \\
\text { Comparison } \\
\text { of NT- } \\
\text { proBNP } \\
\text { levels of } \\
\text { PVCs with } \\
\text { control } \\
\text { group) }\end{array}$ \\
\hline \multicolumn{7}{|l|}{ Gender } \\
\hline Male & 60 & 0.121 & $0.800 \pm 0.08$ & 0.921 & & $0.003^{*}$ \\
\hline Female & 40 & & $0.752 \pm 0.10$ & & & $0.012^{\star}$ \\
\hline \multicolumn{7}{|l|}{ Symptoms } \\
\hline Yes & 73 & $0.00 *$ & $0.811 \pm 0.07$ & 0.699 & $0.232 \pm 0.06$ & $0.002^{*}$ \\
\hline No & 27 & & $0.700 \pm 0.14$ & & & $0.041^{*}$ \\
\hline \multicolumn{7}{|l|}{ PVCs Burden } \\
\hline$<10 \%$ & 33.3 & ns & $0.733 \pm 0.12$ & ns & & $0.039 *$ \\
\hline $10-20 \%$ & 33.3 & & $0.778 \pm 0.09$ & & & $0.020^{*}$ \\
\hline$>20 \%$ & 33.3 & & $0.833 \pm 0.123$ & & & $0.009 *$ \\
\hline \multicolumn{7}{|c|}{$\begin{array}{l}\text { *p value } \leq 0.05 \text { level was considered to be significant. } p \text { values are for comparison among gender, } \\
\text { symptoms and burden for }\end{array}$} \\
\hline \multicolumn{7}{|c|}{$\begin{array}{l}\text { descriptive analysis and NT-proBNP levels. It is also done for NT-proBNP comparison among PVCs } \\
\text { and control group. Data reported as mean } \pm \text { S.E or } n(\%) \text {. }\end{array}$} \\
\hline \multicolumn{7}{|c|}{$\begin{array}{l}\text { PVCs, Premature ventricular contractions; NT-proBNP, N-terminal proBrain natriuretic peptide; ns, non- } \\
\text { significant }\end{array}$} \\
\hline
\end{tabular}

Data analysis of mean NT-proBNP levels among symptomatic and asymptomatic PVCs patients showed that symptomatic PVCs patients were having higher NT-proBNP levels in comparison to asymptomatic patients but the difference was insignificant with $p$ value of 0.699. But the mean NT-proBNP levels in symptomatic as well as asymptomatic patients were significantly higher as compared to the control group with p-values of 0.002 and 0.04 respectively. This shows that even if the NT-proBNP is less in asymptomatic patients, it is still significantly raised than control group. Slightly high levels of NT-pro BNP were observed in male patients as compared to females but the difference was insignificant with $p$ value 
of .921. While comparing the levels of male and female PVCs patients with control group, NT-proBNP levels were significantly higher in males with $p$ value of .003 as well as in females with $p$-value of 0.012 . (table 2)

\section{NT-proBNP and PVCs burden}

There is a significant difference of NT-proBNP levels of control group as compared to PVCs with burden $<10 \%, 10-20 \%$ and more than $20 \%$ with p value of $0.039,0.02$ and 0.009 .The difference of NT-proBNP levels among mild, moderate and frequent PVCs burden were insignificant as shown in table 2.

Figure 1\& 2; shows that there is no correlation of PVCs with NT-proBNP levels in mild and moderate burdens having PVCs $\leq 10 \%$ and $10-20 \%$. While there is a strong positive correlation of PVCs with NTproBNP levels in frequent burden (PVCs $\geq 20 \%$ ) with $r$ value of 0.56 (Fig:3). Bivariate regression analysis was done to examine how well PVCs burden $>20 \%$ can predict the NT-proBNP levels. Scatter plot in fig: 3 shows linear positive correlation with no outliers. The correlation value $r$ is 4.64 and $r^{2}$ is 0.215 ; that is $21.5 \%$ variance in NT-proBNP level was predictable from the level of PVCs burden.

\section{Discussion}

The current study was aimed to determine the levels of NT-proBNP in patients with mild, moderate and high PVCs burdens. Results showed higher NT-proBNP levels in all PVCs burdens (mild, moderate and frequent burdens) in comparison to normal subjects whether they were symptomatic or asymptomatic. This finding can be due to the fact that PVCs cause rapid pacing leading to LV dyssynchrony which results in LV dilation and neurohormonal activation with extracellular remodeling followed by LV dysfunction. This neurohormonal activation triggered by rapid pacing results in release of bioactive peptide, ProBNP which splits into BNP and N-Terminal proBNP. ${ }^{5}$

This study demonstrated the association of PVCs with NT-proBNP which is also supported by a study conducted by Panela de et $\mathrm{al}^{6}$ which showed that NT-proBNP levels fall down after radio frequent ablation of PVCs. The result of the present study is in accordance with that study but that study was conducted on patients with left ventricular dysfunction (Ejection fraction $>50 \%$ ). It has been demonstrated that NT-proBNP levels are found to be raised in patients of left ventricular dysfunction (ejection fraction less than $50 \%$ ) and ventricular dilatation. ${ }^{7}$ In that study high NT-proBNP might be the result of LV dysfunction which dropped down after radiofrequency ablation due to recovery of LV dysfunction. While the present study evaluated the NT-proBNP levels in PVCs patients with preserved LV function (Ejection difference $>50 \%$ ). Muresan $L$ et al ${ }^{8}$ aimed to assess the relation of NT-pro BNP with presence and severity of ventricular arrhythmias in patients with scleroderma. Scleroderma patients with high NT-proBNP in comparison to the patients with low NT-proBNP levels demonstrated presence of ventricular ectopies suggesting PVCs association with NT-proBNP. But that study was conducted on PVCs patients with systemic sclerosis and most of the patients were even having other diseases like pulmonary 
hypertension, pulmonary fibrosis, and impaired spirometry. These conditions might affect the NT-proBNP levels, thus affecting the results. PVCs patients with other diseases such as hypertension, renal disease were excluded from the present study thus minimizing the possibility for NT-proBNP levels to be affected by any other cause/disease.

A study conducted by Xu et al ${ }^{9}$ also found out association of PVCs with higher NT-proBNP levels. It showed normal NT-proBNP levels in patients with mild and moderate burdens but high in frequent burden. That study was aimed to evaluate whether the original site of PVCs and the burden is associated with left ventricular ejection fraction. It showed PVCs originating from tricuspid valve were associated with highest burden and highest NT-proBNP and reduced ejection fraction (even less than $50 \%$ in some patients). A study conducted by Mazurik B et al ${ }^{10}$ established the association of PVCs with NT-proBNP in children. Present study was conducted on adults while children were excluded, but its result coincides with that study and both studies found significantly raised NT-proBNP levels in moderate and frequent PVCs burden in comparison to control group. Present study also found the significant difference between low and control group which is in contrary to that study. A study conducted by Danzin et al ${ }^{11}$ also showed an association of malignant PVCs with NT-proBNP. However, that study showed a significant difference between malignant and benign PVCs in patients with heart failure (ejection fraction $<45 \%$ ) which might have affect the NT-proBNP levels. Another study conducted by yu ho et al ${ }^{12}$ also supported the present findings as high NT-proBNP levels were found to have more ventricular ectopies. But that study was conducted on patients with previous history of $\mathrm{Ml}$ and they were also having heart failure. All patients in that study was having ejection fraction less than $45 \%$ and the higher BNP levels may be due to left ventricular dysfunction. ${ }^{7}$ That study also demonstrated that patients with lower ejection fraction were having higher NT-proBNP as compared to patients with relatively better ejection fraction.

The current study also determined the association of NT-proBNP with low $(<10 \%)$, moderate $(10-20 \%)$ and high ( $\geq 20 \%$ ) ventricular ectopic burden while left ventricular function was preserved in all patients (i.e. Left ventricular ejection fraction was $\geq 50 \%$ ). It showed no correlation of NT-proBNP with PVCs in groups of $<10 \%$ and 10 to $20 \%$ but a significant positive correlation of NT-proBNP was found in a group of frequent PVCs $(>20 \%)$. This could be due to increase in myocardial wall stress in frequent PVCs group as it is well assumed that NTproBNP is released in response to myocardial wall stress which increases in frequent PVCs. ${ }^{13}$ Present study also demonstrated that whether patients were symptomatic or asymptomatic, NT-proBNP were significantly high in both of them as compared to healthy subjects (subjects with no PVCs). Though symptomatic patients have higher NT-proBNP than asymptomatic but the difference was insignificant. A study was conducted by Van Huls Van Taxis et al., (2015) ${ }^{13}$ in which a relation between symptoms, PVCs characteristics and markers of myocardial wall stress (NT-pro BNP and circumferential end systolic wall stress) were evaluated. The study showed that frequent PVCs ( $\geq 24 \%)$ are associated with high NT-proBNP which is consistent with the present study. It also demonstrated high levels of N-Terminal proBNP and circumferential wall stress (CESS) in patients with symptom of fatigue as compared to patients with no fatigue. This finding is in contradiction to present study which shows no significant difference between symptomatic and asymptomatic patients. ${ }^{13}$ 
Sekiguchi et al., (2005) ${ }^{14}$ found out persistently raised NT-proBNP levels in post radiofrequency ablation PVCs patients whom procedure was unsuccessful as compare to the patients with successful radiofrequency ablation. In successful ablation PVCs were successfully eliminated and NT - proBNP fall down significantly. The results of present study are in contradiction to the study conducted by Schey et al ${ }^{15}$ which showed no association of NT-proBNP with PVCs burden. That study was conducted on animals (dogs) and demonstrated that PVCs induced cardiomyopathy (LVEF $<50 \%)$ was present among $11.1 \%, 44 \%$ and $100 \%$ of animals at PVCs burden of $25 \%, 33 \%$ and $50 \%$ respectively, but NT-proBNP or IL 6 were not raised in any of these burdens.

\section{Conclusions}

It is concluded that NT-proBNP were significantly high in all PVCs patients as compared to normal individuals irrespective of symptoms and gender suggesting its significance as a diagnostic tool. It is also concluded that NT-proBNP is found to be high in PVCs patients even if they don't have any symptom. This finding can be significant for its use as a screening tool to identify PVCs patients in a normal population so that the patients with high levels can be advised for further evaluation.

\section{Declarations}

\section{Ethics approval and consent to participate}

The study was approved by ethical review committee of Riphah University. Informed consent was obtained from all subjects or, if subjects are under 18, they were not included in the study.

All the methods were carried out in accordance with relevant guidelines and regulations in the declaration section with ethical statement.

\section{Consent for publication}

Consent was taken by patients for publication

\section{Availability of data and materials}

The datasets used and/or analyzed during the current study available from the corresponding author on reasonable request.

\section{Competing interests}

None 


\section{Funding}

Self

\section{Authors' contributions}

Author S.Ambreen: Conceived, designed, data collection and manuscript writing.

Author HFK: Intellectual input during drafting and designing the project

Author S.Ali: Intellectual input and critical reviewing of the manuscript

Author AH: Interpretation of the data for work and critical review

All authors (S.Ambreen; HFK; S.Ali and AH) reviewed the manuscript.

\section{Acknowledgements}

None

\section{Authors' information (optional)}

Dr. Shazadi Ambreen ${ }^{1}$; Dr. Humaira Fayyaz Khan²; Dr. Shazia Ali³; Dr. Azmat Hayat";

M.B.B.S, M-Phil Physiology; Shifa Tameere-e-Millat University, Islamabad, Pakistan ${ }^{1}$

M.B.B.S, F.C.P.S Physiology; Riphah International University, Islamabad, Pakistan²

M.B.B.S, M-Phil Physiology, Ph.D; Riphah University, Islamabad, Pakistan ${ }^{3}$

MRCP, FRCP, Fellow Electrophysiology, Armed forces institute of cardiology and heart diseases(AFICD), Rawalpindi, Pakistan ${ }^{4}$

\section{References}

1. Berger R, Huelsman M, Strecker K, Bojic A, Moser P, Stanek B, et al. Patients With Chronic Heart Failure. 2002;2392-7.

2. Bettencourt P, Azevedo A, Pimenta J, Friões F, Ferreira S, Ferreira A. N-Terminal-Pro-Brain Natriuretic Peptide Predicts Outcome After Hospital Discharge in Heart Failure Patients. 2010;2168-74.

3. Hall JE, Guyton AC. Guyton and Hall Physiology Review. Guyton and Hall Physiology Review. 2011. 171-190 p. 
4. TADA H, ITO S, SHINBO G, TADOKORO K, ITO I, HASHIMOTO T, et al. Significance and Utility of Plasma Brain Natriuretic Peptide Concentrations in Patients with Idiopathic Ventricular Arrhythmias. Pacing Clin Electrophysiol [Internet]. 2006;29(12):1395-403.

5. Gopinathannair R, Etheridge SP, Marchlinski FE, Spinale FG, Lakkireddy D, Olshansky B. ArrhythmiaInduced Cardiomyopathies Mechanisms, Recognition, and Management. J Am Coll Cardiol. 2015;66(15):1714-28.

6. Penela D, Van Huls Vans Taxis C, Aguinaga L, Fernández-Armenta J, Mont L, Castel MA, et al. Neurohormonal, structural, and functional recovery pattern after premature ventricular complex ablation is independent of structural heart disease status in patients with depressed left ventricular ejection fraction: A prospective multicenter study. J Am Coll Cardiol. 2013;62(13):1195-202.

7. Bay M, Kirk V, Parner J, Hassager C, Nielsen H, Krogsgaard K, et al. NT-proBNP: a new diagnostic screening tool to differentiate between patients with normal and reduced left ventricular systolic function. Heart [Internet]. 2003;89(2):150-4.

8. Muresan L, Petcu A, Muresan C, Rinzis M, Gu-Setu G, Pop D, et al. The role of NT-proBNP in the diagnosis of ventricular arrhythmias in patients with systemic sclerosis. Iran J Public Health. 2017;46(7):906-16.

9. Xu W, Li M, Chen M, Yang B, Wang D, Kong X, et al. Effect of burden and origin sites of premature ventricular contractions on left ventricular function by 7-day Holter monitor. J Biomed Res [Internet]. 2015;29(April):465-74.

10. Mazurek B, Szydłowski L, Giec-Fuglewicz G, Markiewicz-Łoskot G. N-terminal prohormone brain natriuretic peptide-proBNP levels in ventricular arrhythmias in children. Clin Cardiol. 2009;32(12):690-4.

11. Deniz A, Deveci O, Cagliyan CE. Association among tenascin-C and NT-proBNP levels and arrhythmia prevalence in heart failure. 2017;(December).

12. Yu H, Oswald H, Gardiwal A, Lissel C, Klein G. Comparison of N-Terminal Pro-Brain Natriuretic Peptide Versus Electrophysiologic Study for Predicting Future Outcomes in Patients With an Implantable Cardioverter Defibrillator After Myocardial Infarction. Am J Cardiol. 2007;100(4):635-9.

13. Van Huls Van Taxis CFB, Piers SRD, De Riva Silva M, Dekkers OM, Pijnappels DA, Schalij MJ, et al. Fatigue as Presenting Symptom and a High Burden of Premature Ventricular Contractions Are Independently Associated with Increased Ventricular Wall Stress in Patients with Normal Left Ventricular Function. Circ Arrhythmia Electrophysiol. 2015;8(6):1452-9.

14. Sekiguchi Y, Aonuma K, Yamauchi Y, Obayashi T, Niwa A, Hachiya H, et al. Chronic hemodynamic effects after radiofrequency catheter ablation of frequent monomorphic ventricular premature beats. J Cardiovasc Electrophysiol. 2005;16(10):1057-63.

15. Schey KL, Luther JM, Rose KL. HHS Public Access. 2016;13(3):1-21.

\section{Figures}




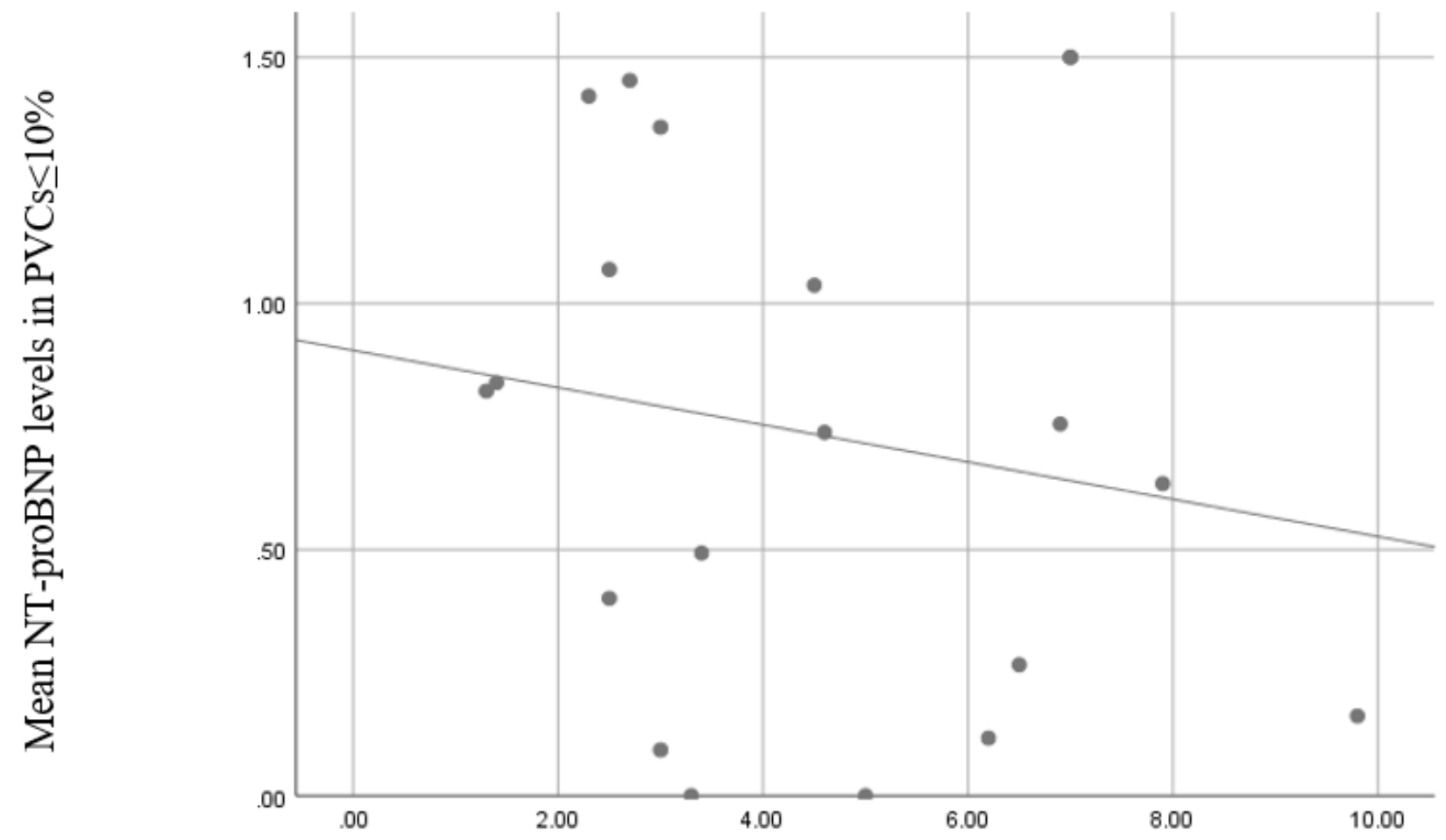

PVCs burden in patients with burden $\leq 10 \%$

\section{Figure 1}

correlation of NT-proBNP with PVCs less 10\% The graph shows that there is no correlation of NT-proBNP with PVCs less than $10 \%$ 


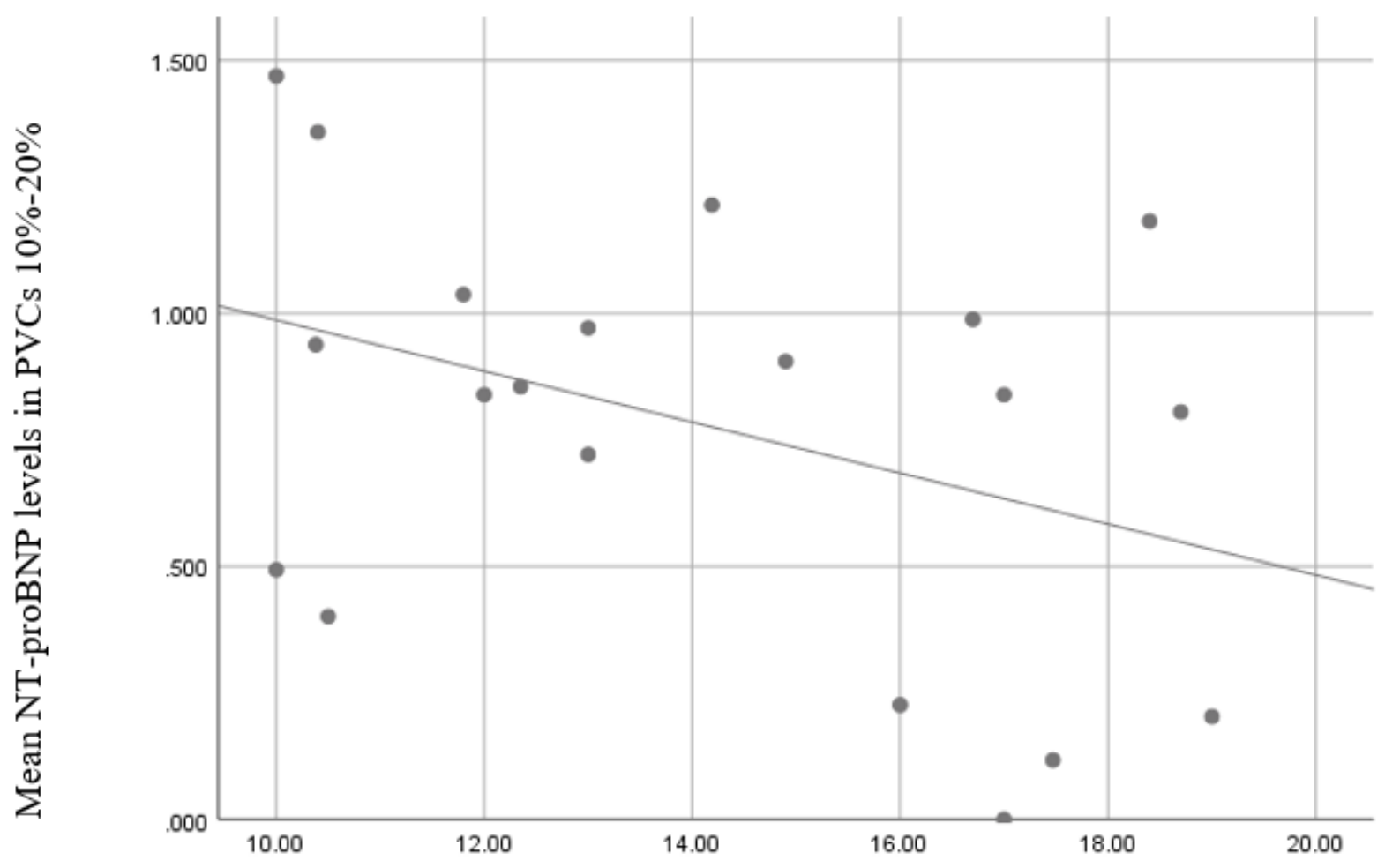

PVCs burden in patients with burden $10-20 \%$

\section{Figure 2}

correlation of NT-proBNP with PVCs $10-20 \%$. The graph shows no correlation of NT pro BNP with PVCs in 10 to $20 \%$. 


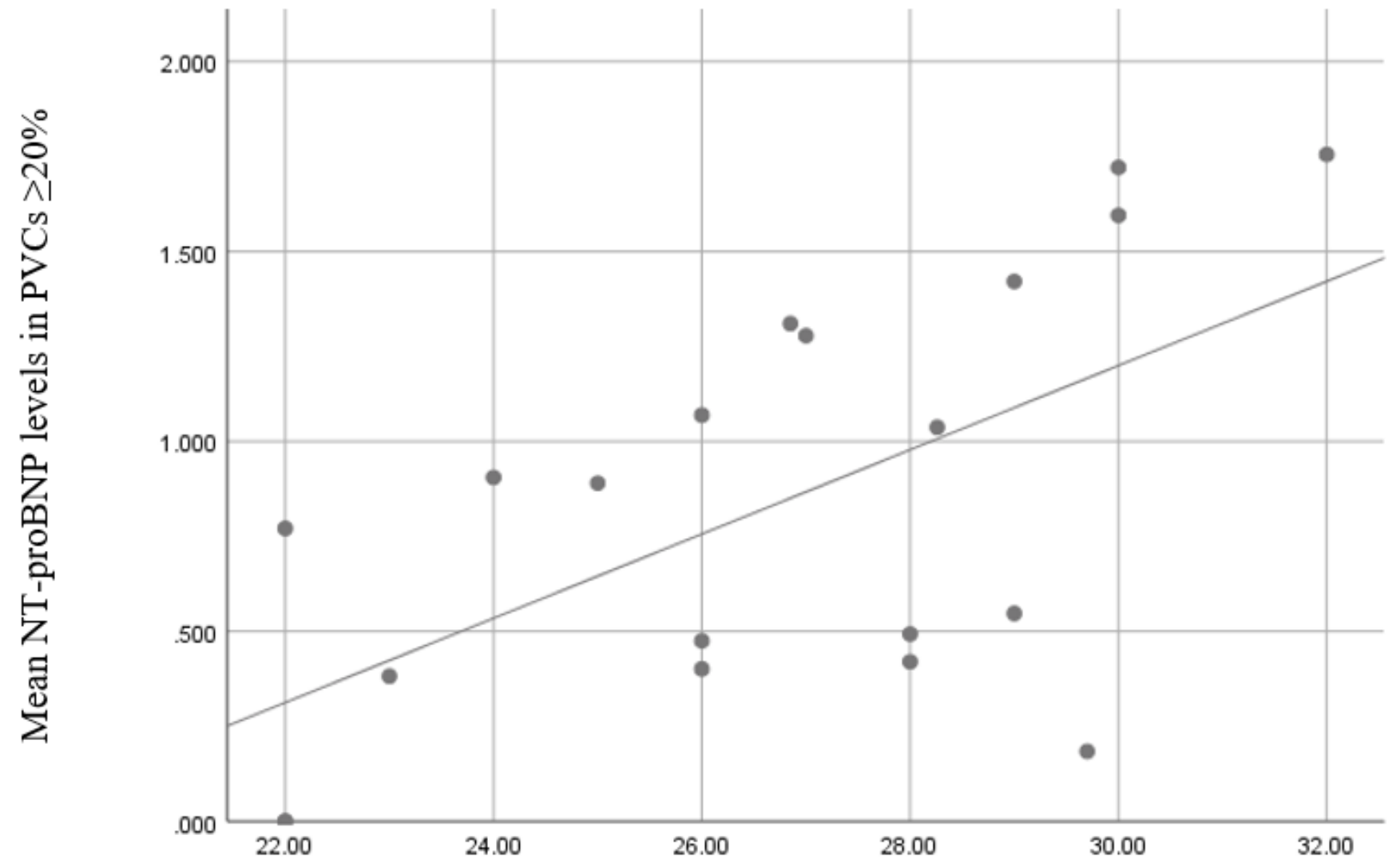

PVCs burden in patients with burden $\geq 20 \%$

\section{Figure 3}

shows correlation of NT-proBNP with PVCs more than $20 \%$ The graph shows positive correlation of NTpro BNP with PVCs more than $20 \%$. 